\title{
Placenta accreta spectrum-a catastrophic situation in obstetrics
}

\author{
Bhabani Pegu, MD, Chitra Thiagaraju, MD, Deepthi Nayak, MD, Murali Subbaiah, MD \\ Department of Obstetrics and Gynecology, Jawaharlal Institute of Postgraduate Medical Education and Research, Puducherry, India
}

Placenta accreta is a significant obstetric complication in which the placenta is completely or focally adherent to the myometrium. The worldwide incidence of placenta accreta spectrum (PAS) is increasing day by day, mostly due to the increasing trends in cesarean section rates. The accurate and timely diagnosis of placenta accreta is important to improve the feto-maternal outcome. Although standard ultrasound is a reliable and primary tool for the diagnosis of placenta accreta, the absence of ultrasound findings does not preclude the diagnosis of placenta accreta. Therefore, clinical evaluation of risk factors is equally essential for the prediction of abnormal placental invasion. Pregnant women with a high impression or established diagnosis of placenta accreta should be managed by a multidisciplinary team in a specialist center. Traditionally, PAS has been managed by an emergency obstetric hysterectomy. Previously, few studies suggested a satisfactory success rate of conservative management in well-chosen cases, whereas few studies recommended delayed hysterectomy to reduce the amount of bleeding. The continuously increasing trends of PAS and the challenges for its routine management are the main motives behind this literature review.

Keywords: Placenta accreta spectrum; Massive hemorrhage; Maternal morbidity; Obstetric hysterectomy

\section{Introduction}

Placenta accreta spectrum (PAS) is a potentially life-threatening obstetric complication. It is characterized by invasive placentation due to the absence of the decidua basalis layer, resulting in placental anchoring villi coming in direct apposition with the myometrium due to a deficient Nitabuch's layer. Different terminology has been used to explain this condition; however, the recent consensus is to include accreta, increta, and percreta in PAS [1]. Depending on the extent of placental invasion, PAS is categorized into placenta accreta and placenta increta. In placenta accreta, placental villi remain attached to the myometrium without invading it, whereas in placenta increta, placental villi penetrate the myometrium and extend up to or beyond the uterine serosa. Placenta accreta is further divided into two types depending on the timing of diagnosis; those diagnosed during the antenatal period by imaging are called expected PAS, and those diagnosed during the intrapartum period with adherent or retained placenta are called unexpected PAS. The diagnosis of PAS will be considered with the presence of one of the following 1) the need for manual removal of the placenta either completely or partially, 2) evidence of gross placental invasion during surgery, 3) ultrasound diagnosis of PAS confirmed during the third stage of labor, and 4) histological confirmation of hysterectomy specimen [2].

Over the past few decades, the rate of cesarean delivery has increased significantly, resulting in a 10-fold increase in the incidence of PAS, and this situation is expected to worsen over the next few years [3]. Despite the advances made in obstetric imaging, multiple studies have shown that a significant number of PAS cases are unpredictable at the time of delivery [4-6]. Management in a multidisciplinary center is

Received: 2020.11.13. Revised: 2021.01.23. Accepted: 2021.03.16. Corresponding author: Bhabani Pegu, MD

Department of Obstetrics and Gynecology, Jawaharlal Institute of Postgraduate Medical Education and Research, Jipmer Campus Rd, Gorimedu, Puducherry 605006, India

E-mail: bpeguamc@gmail.com

https://orcid.org/0000-0002-5475-7856

Articles published in Obstet Gynecol Sci are open-access, distributed under the terms of the Creative Commons Attribution Non-Commercial License (http://creativecommons. org/licenses/by-nc/3.0/) which permits unrestricted non-commercial use, distribution, and reproduction in any medium, provided the original work is properly cited.

Copyright $\odot 2021$ Korean Society of Obstetrics and Gynecology 


\title{
Obstetrics \& Gynecology Science
}

\author{
Vol. 64, No. 3, 2021
}

known to reduce maternal mortality and morbidity in women with PAS, but timely referral and management are challenging in unexpected PAS [7-9]. In 2015, a multicenter study from the Maternal-Fetal Medicine Units Network showed that almost $50 \%$ of reported PAS cases were unexpected. Furthermore, they reported poorer outcomes in patients with expected PAS than those in patients with unexpected PAS; this could be attributed to the referral of patients with a high degree of placental invasion to specialists [10].

The frequency of PAS has progressively increased from 0.8 per 1,000 deliveries in the 1980s to 3 per 1,000 deliveries in the last few years, a phenomenon ascribed to a rising rate of cesarean section globally [11]. The most common and defined risk factor associated with PAS is a previous cesarean section, and the risk is directly proportional to the number of prior cesarean deliveries $[12,13]$. Placenta previa is another significant risk factor for PAS observed in 3\% of women without a previous surgical scar in the uterus. The likelihood of PAS is high in women who have a placenta previa and one or more repeat cesarean deliveries. In such women, the risk of placenta accreta is $3 \%, 11 \%, 40 \%, 61 \%$, and $67 \%$ for the first, second, third, fourth, and fifth or more repeat cesarean deliveries, respectively [12]. Other risk factors include advanced maternal age, multiparty, history of previous uterine surgeries or curettage, and Asherman syndrome $[14,15]$.

Recently, with the advent of in vitro fertilization (IVF) and advancements in cryopreservation techniques, cryopreserved embryo transfer (CET) has been proposed as an additional risk factor for PAS [16-18]. Previous studies have shown that the stimulation protocols for IVF induce morphological and structural changes and disturb the expression of relevant genes in the endometrium; such changes could contribute to abnormal implantation [19]. However, this theory failed to explain the occurrence of PAS in women who underwent frozen-thawed embryo transfer or ovum donation cycles without ovarian stimulation. Another study found that ovarian stimulation cycles, which eventually led to a pregnancy with PAS, had thinner endometrial linings and lower peak serum estrogen (E2) levels when compared to cycles, which led to a pregnancy without PAS. This allowed us to propose a threshold value for serum E2 level at which the risk of placenta accreta was significantly high. They also suggested that the relationship between CET and placenta accreta might be mediated by E2 administration. They found that patients who had been prescribed oral E2 had significantly higher incidence of placenta accreta after CET than those who had been prescribed transdermal or vaginal E2 [20].

PAS is one of the most important causes of maternal mortality and morbidity in the modern obstetrics; it has a mortality as high as $7 \%$ and even higher in underdeveloped countries $[21,22]$. Most of the time, unexpected PAS is diagnosed clinically for the first-time during delivery. Increased maternal morbidity is due to massive intrapartum or postpartum hemorrhage and its sequel, namely, blood transfusions, coagulopathy, sepsis, and multiorgan failure. These situations necessitate medical intervention, and PAS-associated hemorrhage is one of the leading indications for emergency cesarean hysterectomy.

\section{Pathophysiology}

Over the years, various concepts have emerged to explain why and how PAS occurs. The most accepted hypothesis explains the occurrence of PAS in a scarred uterus. Following a uterine scar, there is failure in the normal decidualization of the area is compromised, causing secondary defect in the endometrial-myometrial junction. This defect allows placental anchoring villi, along with the trophoblast, to invade deep into the myometrium [23]. In normal pregnancy, the extent of trophoblastic invasion is regulated by the decidua. In ectopic pregnancy, scarce decidua leads to aggressive implantation into the muscular and serosal layers of the fallopian tube or the abdomen, indicating the important function of decidua as a barrier against abnormal placentation [24,25].

The underlying pathology of PAS is scarred uterus and, in particular, uterine scar secondary to a cesarean section. A recent study has shown that following a cesarean section, uterine vascular resistance is increased, which in turn decreases the blood flow to the endometrium in comparison to that following a normal vaginal delivery [26]. This impaired blood circulation around the scar tissue hampers the vascularization in the scar cascading to permanent focal myometrial degeneration, along with absent or reduced re-epithelialization of the scar area. In the scar area without re-epithelialization, the trophoblast extensively invades beyond the decidua and into the myometrium, reaching up to the underlying blood vessel and nearby structures. Cellular changes in the trophoblast detected in PAS are not a primary defect of trophoblast biology but are possibly secondary to the rare myometrial condi- 


\section{Obstetrics \& Gynecology Science}

Bhabani Pegu, et al. Placenta accreta spectrum disorder

tion leading to excessive invasion into the myometrium [27]. PAS has also been described in first time pregnant women without any previous uterine surgery. The reason for being having PAS in such patient is due to anomalous uterus, other pathology like adenomyosis, dystrophia myotonia, or submucosal uterine fibroids $[28,29]$.

\section{Antenatal diagnosis of PAS}

According to recent studies, a significant number of PAS cases are diagnosed at delivery [4-6]. This may be attributed to the fact that the major known risk factors for PAS such as placenta previa and previous cesarean deliveries are not always present. In addition, even in the presence of known risk factors, the antenatal diagnosis of PAS with less severe placental invasion may be challenging. Prenatal ultrasound, which is the most common diagnostic modality for PAS, is subjective and requires specialized expertise in both performing the examination and interpreting the findings. A previous study also suggested that multiple gestations may make the antenatal diagnosis of PAS more difficult, and we believe that special attention should be paid to placental invasion in multiple pregnancies, especially in the presence of the risk factors for PAS [10]. Therefore, it is very important to recognize various risk factors for PAS during the antenatal period. These high-risk patients would have the opportunity to be assessed more judiciously by using three-dimensional ultrasound with color Doppler imaging and magnetic resonance imaging (MRI). The early antenatal diagnosis of PAS in pregnant women with risk factors is critical to obstetric outcomes because it enables women to have an elective cesarean section and possible hysterectomy and decreases associated complications.

The primary technique for antenatal diagnosis of PAS is obstetric ultrasonography, and the signs of PAS may be present even in the early phase of pregnancy; however, in most women, PAS is recognized in mid- and late pregnancy [30]. The PAS recognition competency of ultrasound depends on the skill, technique, and experience of the observer, and the index of suspicion helps the physician to put forth. Therefore, the findings of normal placental ultrasound imaging do not eliminate the need for a high index of suspicion, especially in pregnant women with established risk factors for PAS. In cases of normally placed placenta, a hypoechoic area is observed between the placental body and the myometrium by ultrasound imaging. In morbidly adherent placenta, the hypoechoic zone between the myometrium and the placenta is absent on ultrasound images. It is very much unexpected to be able to notice a placental mass in the urinary bladder, and that would be the confirmatory point to diagnose true placenta accreta. In Doppler color flow imaging, turbulent lacunar blood flow is the most common finding facilitating the diagnosis of PAS. A marked increase in subplacental vascularity or blood vessels connecting the placenta to uterine margin is expected in this condition. Placental lacunae are considered the most definitive characteristic with high sensitivity and good positive predictive value [31].

MRI is another diagnostic tool for PAS, especially in cases of incomplete or inconclusive ultrasound findings. MRI provides an additional advantage in assessing the depth and lateral extension of abnormal placental invasion [32]. A meta-analysis conducted in 2013 concluded that the overall detection rate of abnormally invasive placenta with ultrasound imaging and MRI was almost the same. They also demonstrated that MRI is superior to ultrasound in detecting posterior PAS, degree of placental invasion, and myometrium lateral extension [33]. A previous study comparing the diagnostic accuracy of ultrasound imaging and MRI found no statistically significant difference in sensitivity or specificity between ultrasound imaging and MRI [34]. A systematic review demonstrated that the validity of MRI for the detection of PAS is reasonably better with a sensitivity of $75 \%$ to $100 \%$ and a specificity of $65 \%$ to $100 \%$ than that of ultrasound imaging. Routine use of MRI is still limited because it is more costly than ultrasonography, is not easily accessible, and requires skill and expertise to interpret the findings. Therefore, MRI is not always a favorable diagnostic tool for the initial assessment of the possible PAS [35].

PAS can occur in the anterior as well as posterior uterine wall. Anterior PAS is a classical invasive placental disorder. The imaging based diagnosis of posterior PAS is more challenging than that of anterior PAS and requires expertise and a high degree of suspicion because the placenta invades into the posterior uterine wall. Antenatal diagnosis of PAS can be performed using ultrasonography and MRI. A recent systematic review on posterior PAS found that almost half of cases affected by posterior PAS did not show the classical ultrasound signs described in anterior PAS cases, and they were not detected by prenatal ultrasound [36]. Overall, 


\title{
Obstetrics \& Gynecology Science
}

\author{
Vol. 64, No. 3, 2021
}

$56.4 \%$ posterior PAS cases were detected by prenatal ultrasound, whereas $46.7 \%$ were diagnosed only at birth. With the advent of MRI, the prenatal detection rate of posterior PAS improved to $73.5 \%$ (95\% confidence interval [CI] 47.892.6), and only about one-fourth of the cases (26.5\%, 95\% $\mathrm{Cl}$ 7.4-52.2) were discovered during cesarean section. Due to diagnostic challenges, the exact incidence of posterior PAS is unclear. Screening for PAS in all women with placenta previa using MRI is not cost effective, and it should be offered to women who are at high risk and show ultrasound signs of placental invasion. Even with this approach, the actual risk of PAS in these women cannot be completely quantified.

\section{Management}

\section{Conservative management of PAS}

In conservative management of PAS, operative management is performed with preservation of the uterus especially to preserve future fertility and to avoid peripartum hysterectomy, PAS-related morbidity, and other consequences. After the delivery of the fetus, the umbilical cord is clamped, ligated, and cut as close as possible to the placenta without attempting to detach the placenta. The use of oxytocin should be discouraged because it may cause incomplete separation of the non-adherent portion of the invasive placenta, leading to profuse bleeding. In PAS, there will be related insinuation of placenta to the neighboring structures is an additional major purpose not to attempt removal of the placenta and make a decision on expected management. An adherent placenta left in situ after the delivery of the fetus may lead to a progressive reduction in blood circulation within the uterus, parametrium, and placenta. In a few days, necrosis of the placental villous tissue is observed, and the placenta gradually detaches from the uterus. Therefore, conservative management aims not only to preserve the uterus but also to limit serious lethal issues related to an attempt of manual removal of the placenta or related to emergency obstetric hysterectomy. Prophylactic antibiotics should be administered for at least 7-10 days after the delivery to avoid infection. Methotrexate has a very limited effect on the placenta during the late phase of pregnancy because it has only a few rapidly dividing cells at that time; therefore, the use of methotrexate is not recommended. Methotrexate is a cytotoxic drug, which may cause significant side effects such as bone mar- row suppression, dizziness, headache, photophobia, hair fall, gastric upset, and dry skin [37].

\section{Management of expected (antenatally diagnosed) PAS}

Patients with expected PAS should be referred to a multidisciplinary center with a medical team experienced in managing PAS patients. Planning the delivery in a highly skilled and experienced maternity center with a well-organized team and the capability to gather additional expertise and resources reduces maternal morbidity and mortality. Counselling sessions to prepare the patient and family members about the possible need for pregnancy termination at any point in time for maternal indications, complications, and obstetric hysterectomy and the possible need for multiple blood transfusions and prolonged Intensive Care Unit (ICU) admission are very important [38]. Before deciding the timing of delivery, the maternal risks and benefits to the fetus or neonate must be weighed. Carrying out a cesarean delivery immediately followed by a peripartum hysterectomy before the onset of labor enhances maternal outcomes although the ideal timing for it remains undefined [39]. Premature delivery may be needed anytime in cases of continuous bleeding per vagina or other obstetric indications such as severe preeclampsia, premature rupture of membranes, fetal distress, or maternal comorbidities. Corticosteroid use is recommended for fetal lung maturation in women with antenatally diagnosed placenta accreta. Conservative management is not suggested beyond 36 weeks of gestation because almost half of the pregnant women with PAS require emergency delivery due to profuse bleeding after 36 weeks of gestation.

The most traditional approach to manage PAS is cesarean section with concurrent obstetric hysterectomy, and the placenta is left untouched inside the uterine cavity after the delivery of the fetus. It is strongly discouraged to forcefully remove the placenta because it may cause massive hemorrhage $[40,41]$. After confirming that the placenta will not separate automatically, many physicians prefer to perform rapid closure of the uterine incision and to proceed with obstetric hysterectomy. Total hysterectomy is essential in most cases of placenta accreta as lower uterine segment or cervical bleeding frequently anticipates supracervical hysterectomy [42]. During the intraoperative period, it is important to closely monitor the vital signs of the patient, volume status, urine output, ongoing blood loss. Ongoing blood loss should 


\section{Obstetrics \& Gynecology Science}

Bhabani Pegu, et al. Placenta accreta spectrum disorder

be restored with the ratio of 1:1:1 to $1: 2: 4$ with the policy of packed red blood cells:fresh frozen plasma (FFP):platelets $[43,44]$. According to a recent meta-analysis, the administration of prophylactic tranexamic acid at the time of delivery after clamping of the cord may reduce the risk of profuse bleeding in patients with PAS [45].

The Triple P procedure was initially developed in 2010 at St George's University Hospital in London to reduce the complications associated with peripartum hysterectomy, such as blood loss, ureteric and bladder injuries, infections, the longterm effect of the presence of prolapse, and the long-term psychological effects of a hysterectomy at a young age. This procedure reduces not only the operative time but also the need for ICU admission and overall hospital stay $[46,47]$. This procedure is performed in three steps: 1) transabdominal ultrasound to locate the placenta and its upper border, 2) making a transverse incision on the upper border of the placenta to deliver the fetus, 3) immediate inflation of the prepositioned balloons for uterine artery occlusion followed by myometrial excision and reconstruction of placental site without separation.

A study was conducted to determine the outcome of the first 50 patients who underwent the Triple P procedure [48]. They observed anterior placenta previa in $58 \%$ of the cases, and an additional $12 \%$ cases had just anterior placenta; $28 \%$ had either posterior or anterior and posterior placenta. The average intraoperative blood loss was $2,318 \mathrm{~mL}$, and vital organs such as bladder and ureteric injury was observed in approximately $2 \%(n=1)$ and $0 \%$, respectively. Duration of hospitalization was 2-8 days, with a median length of stay of 4 days. Three women (6.0\%) developed arterial thrombosis but did not have any long-term squeal. Not a single woman who underwent the Triple P procedure required peripartum hysterectomy. The authors concluded that the Triple P procedure, which is a conservative surgery, reduces morbidity and is an alternative to obstetric hysterectomy. Proper counseling is required for pregnant women with an abnormally invasive placenta during the antenatal period and prior to surgery.

According to Federation of Gynecology and Obstetrics (FIGO), cesarean hysterectomy is associated with a risk of severe maternal morbidity as high as $40-50 \%$. They also reported that maternal mortality rates can be as high as $7 \%$, mainly due to pelvic and vital organ injuries and severe hemorrhage in pregnant women with placenta accrete [49]. The possibilities of severer morbidity and mortality are higher in case of emergency management of placenta accreta compared to those in case of elective management of placenta accreta.

\section{Radiological intervention}

To reduce the morbidity associated with cesarean hysterectomy and uterine preservation, various endovascular procedures have been used in which interventional radiologists play a vital role. Prior to elective cesarean hysterectomy, a prophylactic balloon catheter is placed in the internal iliac arteries, resulting in a significant reduction in intraoperative blood loss, transfusion requirements, and postoperative morbidity [50-52]. One of the limitations of this technique is that it only provides temporary hemostasis. Another endovascular intervention is the infrarenal aortic balloon catheter, which can also be used prior to cesarean hysterectomy. Various studies have found it to be a safe and effective procedure, with low intraoperative blood loss, and its short fluoroscopy duration reduces radiation exposure to the fetus $[53,54]$. Uterine artery embolization (UAE), along with uterine sparing techniques, has been used both intraoperatively and postoperatively to decrease hemorrhage during cesarean hysterectomy. In patients with placenta accreta, UAE has been found to aid placental resorption [55]. It can be used prophylactically or in emergency settings.

\section{Management of unexpected (accidentally detected during the intrapartum period) PAS}

Most PAS cases are unexpectedly detected during the intrapartum period, at the time of either vaginal delivery or cesarean section. After the delivery of the fetus, spontaneous separation of the placenta or attempts to remove the placenta fails in such a condition. If placenta accreta is suspected depending on the uterine appearance during cesarean section, the case should be paused for a transient period until skilled and experienced surgeon arrives. In addition, the anesthesia team should be informed regarding the intraoperative findings and should be asked to alter the type of anesthesia from regional to general, and blood products need to be arranged immediately. If such a facility is not available in that 


\title{
Obstetrics \& Gynecology Science
}

\author{
Vol. 64, No. 3, 2021
}

particular center, contemplation for stabilization and transfer to a higher center is mandatory. Once the diagnosis of PAS is confirmed, rapid closure of the uterine incision should be performed, and obstetric hysterectomy should be performed as judiciously as possible.

Delayed hysterectomy is considered when placental adherence is diagnosed accidentally during cesarean section without adequate preparation/facility for hysterectomy and counseling. In such circumstances, if there is no active bleeding and the vital signs of the patient are stable, the uterus and abdomen can be closed rapidly before transferring the patient to a center with a multidisciplinary specialist team. Secondary hysterectomy can be considered 6-10 weeks after the delivery [56]. This option is also applicable for patients who delivered vaginally; in these patients, the umbilical cord is ligated and cut as short as possible if PAS is highly suspected using one of the imaging tools. The benefit of performing delayed hysterectomy over emergency hysterectomy is to reduce blood loss and accidental injury to the nearby vital organs.

\section{Postoperative considerations and management}

For intensive monitoring of hemodynamic status and early postoperative care, the patient should be transferred to the ICU immediately after the surgery. Close and frequent communication between the operative team and the immediate postoperative care team are strongly encouraged. Vital sign stability, any ongoing bleeding from the abdominal and pelvic cavity, fluid and electrolyte balance, any signs of multiple organ failure, and the need for any other supportive therapy should to be closely monitored.

The patient's family members should be informed regularly about the patient's condition. In the postoperative period, the patient needs to be observed for the signs and symptoms of Sheehan's syndrome, which could lead to lactation failure in addition to other presentations.

\section{Conclusion}

PAS is associated with significant maternal morbidity and mortality. The number of PAS cases has been increasing in recent years, and it is likely to increase further in the coming years due to the increasing trend of cesarean sections and assisted reproductive techniques. Clinical assessment of risk factors for placenta accreta and prenatal imaging may help guide the diagnosis. Antenatal diagnosis is the key to improving maternal and fetal outcomes and reducing morbidity and mortality. The most common traditional management of placenta accreta is obstetric hysterectomy, which should be performed by the most skilled and experienced surgeons in a center with a multidisciplinary team, resources, and adequate blood products. The conservative management of placenta accreta should be considered only in carefully selected cases.

\section{Conflict of interest}

No potential conflict of interest relevant to this article was reported.

\section{Ethical approval}

This study does not require approval of the Institutional Review Board because no patient data is contained in this article. The study was performed in accordance with the principles of the Declaration of Helsinki.

\section{Patient consent}

Written informed consent and the use of images from patients are not required for the publication.

\section{Funding information}

None.

\section{References}

1. Jauniaux E, Chantraine F, Silver RM, Langhoff-Roos J, for the FIGO Placenta Accreta Diagnosis and Management Expert Consensus Panel. FIGO consensus guidelines on placenta accreta spectrum disorders: epidemiology. Int J 


\section{Obstetrics \& Gynecology Science}

Bhabani Pegu, et al. Placenta accreta spectrum disorder

Gynecol Obstet 2018;140:265-73.

2. Mittal P, Suri J, Pruthi N, Pandey D, Bharti R. Comparison of placenta accreta spectrum disorders diagnosed in intrapartum and antepartum period-a three year experience in a tertiary referral unit of India. Eur J Obstet Gynecol Reprod Biol 2019;236:41-5.

3. Solheim KN, Esakoff TF, Little SE, Cheng YW, Sparks TN, Caughey $A B$. The effect of cesarean delivery rates on the future incidence of placenta previa, placenta accreta, and maternal mortality. J Matern Fetal Neonatal Med 2011;24:1341-6.

4. Fitzpatrick KE, Sellers S, Spark P, Kurinczuk JJ, Brocklehurst $\mathrm{P}$, Knight $\mathrm{M}$. The management and outcomes of placenta accreta, increta, and percreta in the UK: a population-based descriptive study. BJOG 2014;121:6271.

5. Thurn L, Lindqvist PG, Jakobsson $M$, Colmorn LB, Klungsoyr $\mathrm{K}$, Bjarnadóttir Rl, et al. Abnormally invasive placenta-prevalence, risk factors and antenatal suspicion: results from a large population-based pregnancy cohort study in the Nordic countries. BJOG 2016;123:1348-55.

6. Bowman ZS, Eller AG, Kennedy AM, Richards DS, Winter TC 3rd, Woodward PJ, et al. Accuracy of ultrasound for the prediction of placenta accreta. Am J Obstet Gynecol 2014;211:177.e1-7.

7. Eller AG, Bennett MA, Sharshiner M, Masheter C, Soisson $A P$, Dodson $M$, et al. Maternal morbidity in cases of placenta accreta managed by a multidisciplinary care team compared with standard obstetric care. Obstet Gynecol 2011;117(2 Pt 1):331-7.

8. Shamshirsaz AA, Fox KA, Erfani H, Clark SL, Salmanian $B$, Baker BW, et al. Multidisciplinary team learning in the management of the morbidly adherent placenta: outcome improvements over time. Am J Obstet Gynecol 2017;216:612.e1-5.

9. Shamshirsaz AA, Fox KA, Erfani H, Clark SL, Shamshirsaz $A A$, Nassr AA, et al. Outcomes of planned compared with urgent deliveries using a multidisciplinary team approach for morbidly adherent placenta. Obstet Gynecol 2018;131:234-41.

10. Bailit JL, Grobman WA, Rice MM, Reddy UM, Wapner RJ, Varner MW, et al. Morbidly adherent placenta treatments and outcomes. Obstet Gynecol 2015;125:683-9.

11. Higgins MF, Monteith C, Foley M, O'Herlihy C. Real increasing incidence of hysterectomy for placenta accreta following previous caesarean section. Eur J Obstet Gynecol Reprod Biol 2013;171:54-6.

12. Silver RM, Landon MB, Rouse DJ, Leveno KJ, Spong CY, Thom EA, et al. Maternal morbidity associated with multiple repeat cesarean deliveries. Obstet Gynecol 2006;107:1226-32.

13. Miller DA, Chollet JA, Goodwin TM. Clinical risk factors for placenta previa-placenta accreta. Am J Obstet Gynecol 1997;177:210-4.

14. Garmi G, Salim R. Epidemiology, eitiology, diagnosis and management of placenta accreta. Obstet Gynecol Int 2012;2012:873929.

15. Baldwin HJ, Patterson JA, Nippita TA, Torvaldsen S, Ibiebele I, Simpson JM, et al. Antecedents of abnormally invasive placenta in primiparous women: risk associated with gynecologic procedures. Obstet Gynecol 2018; 131:227-33.

16. Fitzpatrick KE, Sellers S, Spark P, Kurinczuk JJ, Brocklehurst $P$, Knight $M$. Incidence and risk factors for placenta accreta/increta/percreta in the UK: a national casecontrol study. PLoS One 2012;7:e52893.

17. Hayashi M, Nakai A, Satoh S, Matsuda Y. Adverse obstetric and perinatal outcomes of singleton pregnancies may be related to maternal factors associated with infertility rather than the type of assisted reproductive technology procedure used. Fertil Steril 2012;98:922-8.

18. Ishihara O, Araki R, Kuwahara A, Itakura A, Saito H, Adamson GD. Impact of frozen-thawed single-blastocyst transfer on maternal and neonatal outcome: an analysis of 277,042 single-embryo transfer cycles from 2008 to 2010 in Japan. Fertil Steril 2014;101:128-33.

19. Horcajadas JA, Riesewijk A, Polman J, van Os R, Pellicer A, Mosselman $S$, et al. Effect of controlled ovarian hyperstimulation in IVF on endometrial gene expression profiles. Mol Hum Reprod 2005;11:195-205.

20. Kaser DJ, Melamed A, Bormann CL, Myers DE, Missmer SA, Walsh BW, et al. Cryopreserved embryo transfer is an independent risk factor for placenta accreta. Fertil Steril 2015;103:1176-84.e2.

21. O'Brien JM, Barton JR, Donaldson ES. The management of placenta percreta: conservative and operative strategies. Am J Obstet Gynecol 1996;175:1632-8.

22. Ajah LO, Eze MI, Dim CC, Ezegwui HU, Nkwo PO, Eluke CC, et al. Placenta percreta in a booked multiparous woman with minimal risk factors and challenges of 


\section{Obstetrics \& Gynecology Science}

Vol. 64, No. 3, 2021

management in a low resource setting. Niger J Med 2012;21:359-60.

23. Jauniaux E, Jurkovic D. Placenta accreta: pathogenesis of a 20th century iatrogenic uterine disease. Placenta 2012;33:244-51.

24. Randall S, Buckley CH, Fox H. Placentation in the fallopian tube. Int I Gynecol Pathol 1987;6:132-9.

25. Godyn JJ, Hazra A, Gulli VM. Subperitoneal placenta accreta succenturiate in the case of a successful nearterm extrauterine abdominal pregnancy. Hum Pathol 2005;36:922-6.

26. Flo K, Widnes C, Vårtun Å, Acharya G. Blood flow to the scarred gravid uterus at 22-24 weeks of gestation. BJOG 2014;121:210-5.

27. Jauniaux E, Collins S, Burton GJ. Placenta accreta spectrum: pathophysiology and evidence-based anatomy for prenatal ultrasound imaging. Am J Obstet Gynecol 2018;218:75-87.

28. Fox H. Placenta accreta: 1945-1969. Obstet Gynecol Surv 1972;27:475-90.

29. Benirschke K, Burton GJ, Baergen RN. Pathology of the human placenta, 6th ed. Berlin: Springer-Verlag; 2012.

30. Cahill AG, Beigi R, Heine RP, Silver RM, Wax JR, American College of Obstetricians and Gynecologists. Placenta Accreta Spectrum. Am J Obstet Gynecol 2018;219:B216.

31. Baughman WC, Corteville JE, Shah RR. Placenta accreta: spectrum of US and MR imaging findings. Radiographics 2008;28:1905-16.

32. Jauniaux E, Alfirevic Z, Bhide AG, Belfort MA, Burton GJ, Collins SL, et al. Placenta praevia and placenta accreta: diagnosis and management: green-top guideline no. 27a. BJOG 2019;126:e1-48.

33. D'Antonio F, lacovella C, Bhide A. Prenatal identification of invasive placentation using ultrasound: systematic review and meta-analysis. Ultrasound Obstet Gynecol 2013:42:509-17.

34. D'Antonio F, lacovella C, Palacios-Jaraquemada J, Bruno $\mathrm{CH}$, Manzoli L, Bhide A. Prenatal identification of invasive placentation using magnetic resonance imaging: systematic review and meta-analysis. Ultrasound Obstet Gynecol 2014;44:8-16.

35. Reddy YS, Y A, Ramalakshmi BA, Kumar BD. Lead and trace element levels in placenta, maternal and cord blood: a cross-sectional pilot study. J Obstet Gynaecol
Res 2014;40:2184-90.

36. Tinari S, Buca D, Cali G, Timor-Tritsch I, PalaciosJaraquemada J, Rizzo G, et al. Systematic review and meta-analysis of posterior placenta accreta spectrum disorders: risk factors, histopathology and diagnostic accuracy. Ultrasound Obstet Gynecol. 2020 Aug 25 [Epub]. https://doi.org/10.1002/uog.22183.

37. Meyer NP, Ward GH, Chandraharan E. Conservative approach to the management of morbidly adherent placentae. Ceylon Med J 2012;57:36-9.

38. American College of Obstetricians and Gynecologists. ACOG practice bulletin no. 135: second-trimester abortion. Obstet Gynecol 2013;121:1394-406.

39. Silver RM, Barbour KD. Placenta accreta spectrum: accreta, increta, and percreta. Obstet Gynecol Clin North Am 2015;42:381-402.

40. Eller AG, Porter TF, Soisson P, Silver RM. Optimal management strategies for placenta accrete. BJOG 2009;116:648-54.

41. Warshak CR, Ramos GA, Eskander R, Benirschke K, Saenz CC, Kelly TF, et al. Effect of predelivery diagnosis in 99 consecutive cases of placenta accrete. Obstet Gynecol 2010;115:65-9.

42. Clark SL, Phelan JP, Yeh SY, Bruce SR, Paul RH. Hypogastric artery ligation for obstetric hemorrhage. Obstet Gynecol 1985;66:353-6.

43. Panigrahi AK, Yeaton-Massey A, Bakhtary S, Andrews J, Lyell DJ, Butwick AJ, et al. A standardized approach for transfusion medicine support in patient with morbidly adherent placenta. Anesth Analg 2017;125:603-8.

44. Committee on Practice Bulletins-Obstetrics. Practice bulletin no. 183: postpartum hemorrhage. Obstet Gynecol 2017; 130:e168-86.

45. Simonazzi G, Bisulli M, Saccone G, Moro E, Marshall A, Berghella $V$. Tranexamic acid for preventing postpartum blood loss after cesarean delivery: a systematic review and meta-analysis of randomized controlled trials. Acta Obstet Gynecol Scand 2016;95:28-37.

46. Chandraharan E, Rao S, Belli AM, Arulkumaran S. The triple-P procedure as a conservative surgical alternative to peripartum hysterectomy for placenta percreta. Int J Gynaecol Obstet 2012;117:191-4.

47. Teixidor Viñas M, Belli AM, Arulkumaran S, Chandraharan E. Prevention of postpartum hemorrhage and hysterectomy in patients with morbidly adherent placenta: 


\section{Obstetrics \& Gynecology Science}

Bhabani Pegu, et al. Placenta accreta spectrum disorder

a cohort study comparing outcomes before and after introduction of the triple-P procedure. Ultrasound Obstet Gynecol 2015;46:350-5.

48. Pinas-Carrillo A, Bhide A, Moore J, Hartopp R, Belli AM, Arulkumaran $S$, et al. Outcomes of the first 50 patients with abnormally invasive placenta managed using the "triple P procedure" conservative surgical approach. Int J Gynaecol Obstet 2020;148:65-71.

49. Sentilhes L, Kayem G, Chandraharan E, Palacios-Jaraquemada J, Jauniaux E. FIGO consensus guidelines on placenta accreta spectrum disorders: conservative management. Int J Gynecol Obstet 2018;140:291-8.

50. Ballas J, Hull AD, Saenz C, Warshak CR, Roberts AC, Resnik RR, et al. Preoperative intravascular balloon catheters and surgical outcomes in pregnancies complicated by placenta accreta: a management paradox. Am J Obstet Gynecol 2012;207:216.e1-5.

51. Tan CH, Tay KH, Sheah K, Kwek K, Wong K, Tan HK, et al. Perioperative endovascular internal iliac artery occlusion balloon placement in management of placenta accreta. AJR Am J Roentgenol 2007;189:1158-63.

52. Salim R, Chulski A, Romano S, Garmi G, Rudin M, Sha- lev E. Precesarean prophylactic balloon catheters for suspected placenta accreta: a randomized controlled trial. Obstet Gynecol 2015;126:1022-8.

53. Wu Q, Liu Z, Zhao X, Liu C, Wang Y, Chu Q, et al. Outcome of pregnancies after balloon occlusion of the infrarenal abdominal aorta during caesarean in 230 patients with placenta praevia accreta. Cardiovasc Intervent Radiol 2016;39:1573-9.

54. Wang $Y L$, Duan XH, Han XW, Wang L, Zhao XL, Chen $Z M$, et al. Comparison of temporary abdominal aortic occlusion with internal iliac artery occlusion for patients with placenta accreta - a non-randomised prospective study. Vasa 2017:46:53-7.

55. Fox KA, Shamshirsaz AA, Carusi D, Secord AA, Lee P, Turan OM, et al. Conservative management of morbidly adherent placenta: expert review. Am J Obstet Gynecol 2015;213:755-60.

56. Sawada M, Matsuzaki S, Mimura K, Kumasawa K, Endo $M$, Kimura T. Successful conservative management of placenta percreta: investigation by serial magnetic resonance imaging of the clinical course and a literature review. J Obstet Gynaecol Res 2016;42:1858-63. 